\title{
How robust is rational choice?
}

Felix J. Nitsch and Tobias Kalenscher

Comparative Psychology, Heinrich-Heine-University Düsseldorf, Germany

\begin{abstract}
Author Note
${ }^{*}$ Corresponding author. Felix J. Nitsch, Comparative Psychology, Heinrich-Heine-University Düsseldorf, 40225 Düsseldorf, Germany. Email: felix.nitsch@hhu.de

The research was supported by a grant from the Deutsche Forschungsgemeinschaft (Bonn, Germany) to Tobias Kalenscher (grant-ID: DFG-KA 2675/7-1). We want to thank Alina Keßler, Paul Kramer, and Marie Ludes for their assistance with the literature research and Paula Marie Klug for her assistance with polishing the p-curve disclosure table.
\end{abstract}




\begin{abstract}
Neoclassic economic choice theory assumes that decision-makers make choices as if they were rational agents. This assumption has been critically challenged over the last decades, yet systematic aggregation of evidence beyond single experiments is still surprisingly sparse. Here, we asked how robust choice-consistency, as a proxy for rationality, is to endogenous and exogeneous factors. To this end, we conducted a systematic quantitative literature research, reviewing 5327 articles, identifying 44 as relevant that contained hypothesis tests on possible influence factors of choice-consistency. To assess the evidential value of any effect of such influence factors on choiceconsistency, we conducted a robust $\mathrm{p}$-curve analysis. Our results indicate that choice-consistency is affected by endogenous or exogeneous factors. This result holds for multiple testing procedures and a robustness check. However, due to the breadth of the contemporary research agenda, the lack of replications and the unavailability of original data in the field of choice-consistency, it is currently not possible to draw meaningful conclusions regarding specific influence factors. Despite this lack of specificity, our results implicate that people's decisions might be a noisier and more biased indicator of their underlying preferences than previously thought. Hence, we provide systematic evidence for the wide-spread belief that rationality cannot be assumed unconditionally.
\end{abstract}

\title{
Introduction
}

Which career should I pursue? Which party should I vote for in the 2021 German federal elections? Decisions shape human lives and society, arguably, like no other psychological entity. The question of how to make good or rational decisions has puzzled philosophers, economists, and psychologists for centuries until today.

The predominant theory of rational choice is subjective utility maximization (SUM). In a nutshell, decision-makers are assumed to rank order all available choice options according to their subjective utility and select the one ranked highest. However, to this day it is not completely clear how, if at all, subjective utility values are represented (neuro-)psychologically (Hayden \& Niv, 2020). 
If treated as an non-psychological entity, subjective utility cannot be measured directly (Gul \& Pesendorfer, 2008). Given this measurement problem, it is non-trivial to evaluate whether a specific decision was made for the option with maximum subjective utility and, therefore, was a good decision. Contemporary research generally uses tests on the choice structure of preferences. A milestone of $20^{\text {th }}$ century economics lies in the proof that SUM requires consistency of the choice structure (Afriat, 1973; Houthakker, 1950; Samuelson, 1938; Varian, 1982): the choice of an option 1 over another, less expensive option 2 implies higher subjective utility of option 1.

Neoclassic rational choice theory has been criticized on several grounds (e.g. Arkes et al., 2016; Cason \& Plott, 2014). However, a surge of recent publications pictures rationality as surprisingly robust (Nitsch \& Kalenscher, 2020). A systematic and quantitative integration of these conflicting lines of research is currently missing, which we seek to provide here.

Specifically, we posed the research question of how robust choice-consistency, as a proxy for rationality, is to endogenous and exogeneous factors, such as age, drugs, education, emotions, financial status, intelligence, neurological status, personality, sex and gender, sleep deprivation, or stress. Importantly, we were interested in the influence of factors that may vary between participants, but are assumed to be constant within participants during the period of observation, so that covert responsive preference adaptation can be excluded as underlying source of overt inconsistency.

\section{Results \& Discussion}

To answer how robust choice-consistency, as a proxy for rationality, is to endogenous and exogeneous factors, we conducted a systematic quantitative review of 5327 articles, identifying 44 research articles that contained hypothesis tests which addressed the influence of at least one of the predefined (see above), or related factors. To quantitatively aggregate the evidence in the literature, we conducted a P-curve analysis. P-curve analyses test how reliably a given effect is replicated in the literature by quantifying the evidential value and statistical power (Simonsohn et al., 2014, 2015). 
The rationale behind this analysis is that, under the null hypothesis of no effect of endogenous or exogeneous factors on choice-consistency, the distribution of $p$-values of the effect of any such factor on choice-consistency in the published literature should follow a uniform distribution. On the other hand, in the presence of a true effect, the distribution of p-values should be positively and exponentially skewed. To account for the file drawer problem in scientific publications (Ioannidis et al., 2014; Nosek et al., 2013), the analysis only considers significant $p$-values ( $p<.05)$. Hence, to test our hypothesis, we considered independent significance tests for which the full test-statistic was reported or could be recalculated $(\mathrm{N}=29)$ and for which results indicated a significant effect $(\mathrm{p}<.05$; $\mathrm{N}=21$ ).

Descriptively, 17 of all 21 significant $p$-values (81\%) fell in the lower half of the range of significant $p$-values ( $p<0.025$; see figure 1 ). In line with this, our P-curve analysis indicated evidential value for that choice-consistency is affected by endogenous or exogeneous factors (see table 1; binomial test: $p=.0036$; Stouffer method: $Z=-10.97, p<.0001$ for full $p$-curve and $Z=-11.42, p<.0001$ for half $\mathrm{p}$-curve). Further, we find no evidence that studies' evidential value is inadequate (see table 1; binomial test: $p=.9028$; Stouffer method: $Z=7.08, p>.9999$ for full $p$-curve and $Z=11.04, p>.9999$ for half $\mathrm{p}$-curve). The statistical power estimate of the included studies amounted to $98 \%(90 \%-\mathrm{Cl}$ : 94\%-99\%).

Overall, it is important to point out that the breadth of the search for influence factors (roughly more than 20 different influence factors in 44 articles; see figure 2 for a bibliographic analysis) stands in contrast to the severe lack of replications. Hence, it is currently not possible to draw meaningful conclusions regarding specific influence factors. Future research in the field should be careful to not only focus on finding novel influence factors but also validate the replicability of findings and find a common conceptual structure. In line with that, we should strive to rigorously make original data available to enable more efficient methods of data accumulation than the $p$ curve analysis utilized here. 
The robustness of rationality has been a long-standing question in economics and psychology. Our results show that rationality cannot just be assumed for all decision-makers under all circumstances but instead endogenous and exogenous factors must be considered, even if these factors remain unchanged during the time period of observation. However, while our analysis unequivocally reveals that people's decisions might be a noisier and more biased indicator of their underlying preferences than previously thought, it remains unclear what it is exactly that makes them leave the path of rationality.

\section{References}

Afriat, S. N. (1973). On a system of inequalities in demand analysis: An extension of the classical method. International Economic Review, 460-472. https://doi.org/10.2307/2525934

Arkes, H. R., Gigerenzer, G., \& Hertwig, R. (2016). How bad is incoherence? Decision, 3(1), 20-39. https://doi.org/10.1037/dec0000043

Cason, T. N., \& Plott, C. R. (2014). Misconceptions and Game Form Recognition: Challenges to Theories of Revealed Preference and Framing. Journal of Political Economy, 122(6), 12351270. https://doi.org/10.1086/677254

Gul, F., \& Pesendorfer, W. (2008). The case for mindless economics. The Foundations of Positive and Normative Economics: A Handbook, 1, 3-42.

Hayden, B., \& Niv, Y. (2020). The case against economic values in the brain [Preprint]. PsyArXiv. https://doi.org/10.31234/osf.io/7hgup

Houthakker, H. S. (1950). Revealed preference and the utility function. Economica, 17(66), 159-174. https://doi.org/10.2307/2549382

Ioannidis, J. P. A., Munafò, M. R., Fusar-Poli, P., Nosek, B. A., \& David, S. P. (2014). Publication and other reporting biases in cognitive sciences: Detection, prevalence, and prevention. Trends in Cognitive Sciences, 18(5), 235-241. https://doi.org/10.1016/j.tics.2014.02.010 
Nitsch, F. J., \& Kalenscher, T. (2020). Keeping a cool head at all times. What determines choice consistency? [Preprint]. PsyArXiv. https://doi.org/10.31234/osf.io/etyhx

Nosek, B., Spies, J. R., \& Motyl, M. (2013). Scientific Utopia: II. Restructuring Incentives and Practices to Promote Truth Over Publishability. Perspectives on Psychological Science, 7(6), 615-631. https://doi.org/DOI: 10.1177/1745691612459058

Samuelson, P. A. (1938). A note on the pure theory of consumer's behaviour. Economica, 5(17), 6171.

Simonsohn, U., Nelson, L. D., \& Simmons, J. P. (2014). P-curve: A key to the file-drawer. Journal of Experimental Psychology: General, 143(2), 534-547. https://doi.org/10.1037/a0033242

Simonsohn, U., Simmons, J. P., \& Nelson, L. D. (2015). Better P-curves: Making P-curve analysis more robust to errors, fraud, and ambitious P-hacking, a Reply to Ulrich and Miller (2015). Journal of Experimental Psychology: General, 144(6), 1146-1152.

https://doi.org/10.1037/xge0000104

Varian, H. R. (1982). The nonparametric approach to demand analysis. Econometrica: Journal of the Econometric Society, 945-973. https://doi.org/10.2307/1912771 


\section{Figure 1}

Visualization of the empirical P-curve compared to 2 benchmarks

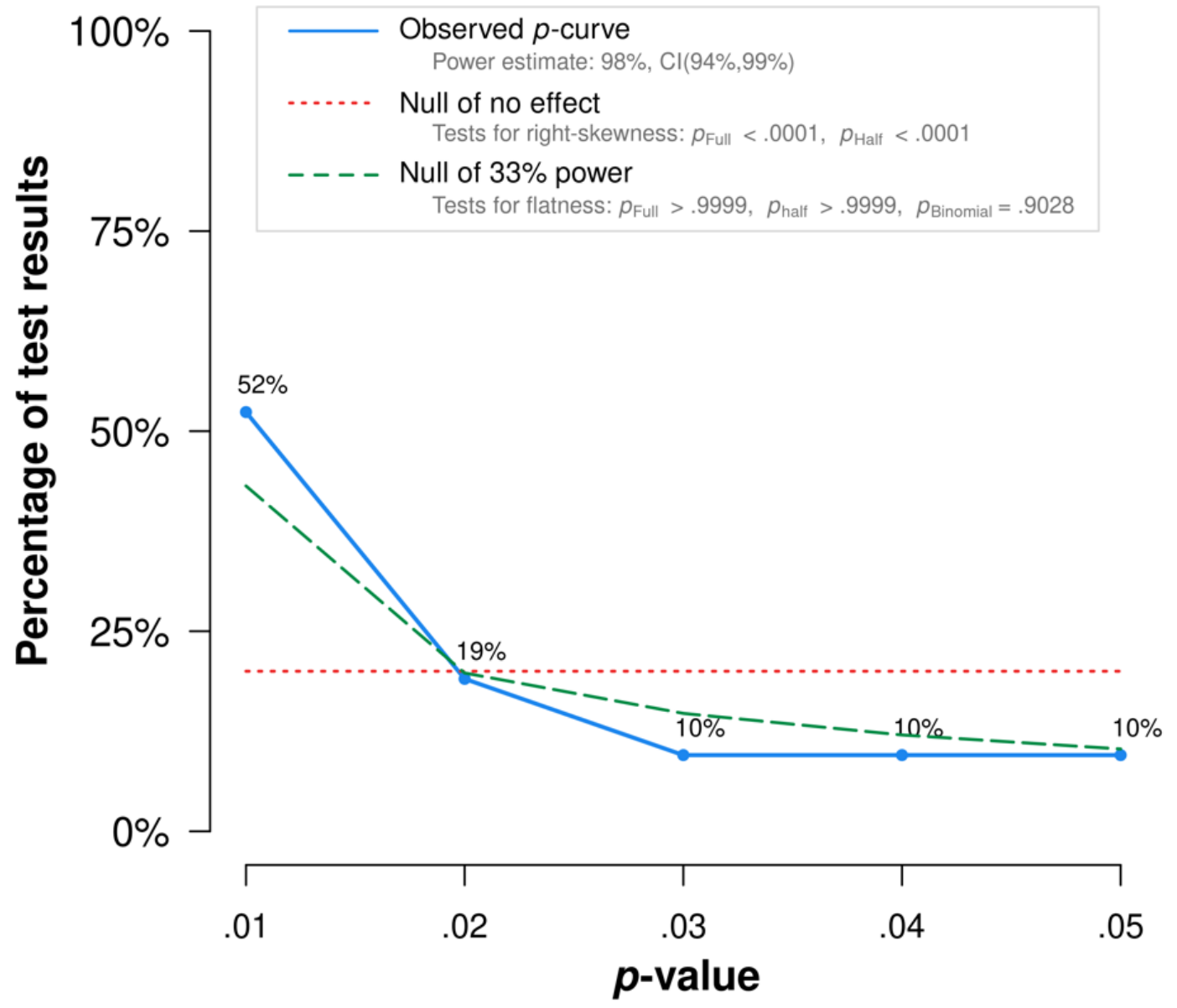

Note: The observed $p$-curve includes 21 statistically significant $(p<.05)$ results, of which 17 are $p<.025$. There were 8 additional results entered but excluded from $p$-curve because they were $p>.05$. 


\section{Figure 2}

Rank Correlation Heatmap of Article Co-Citations

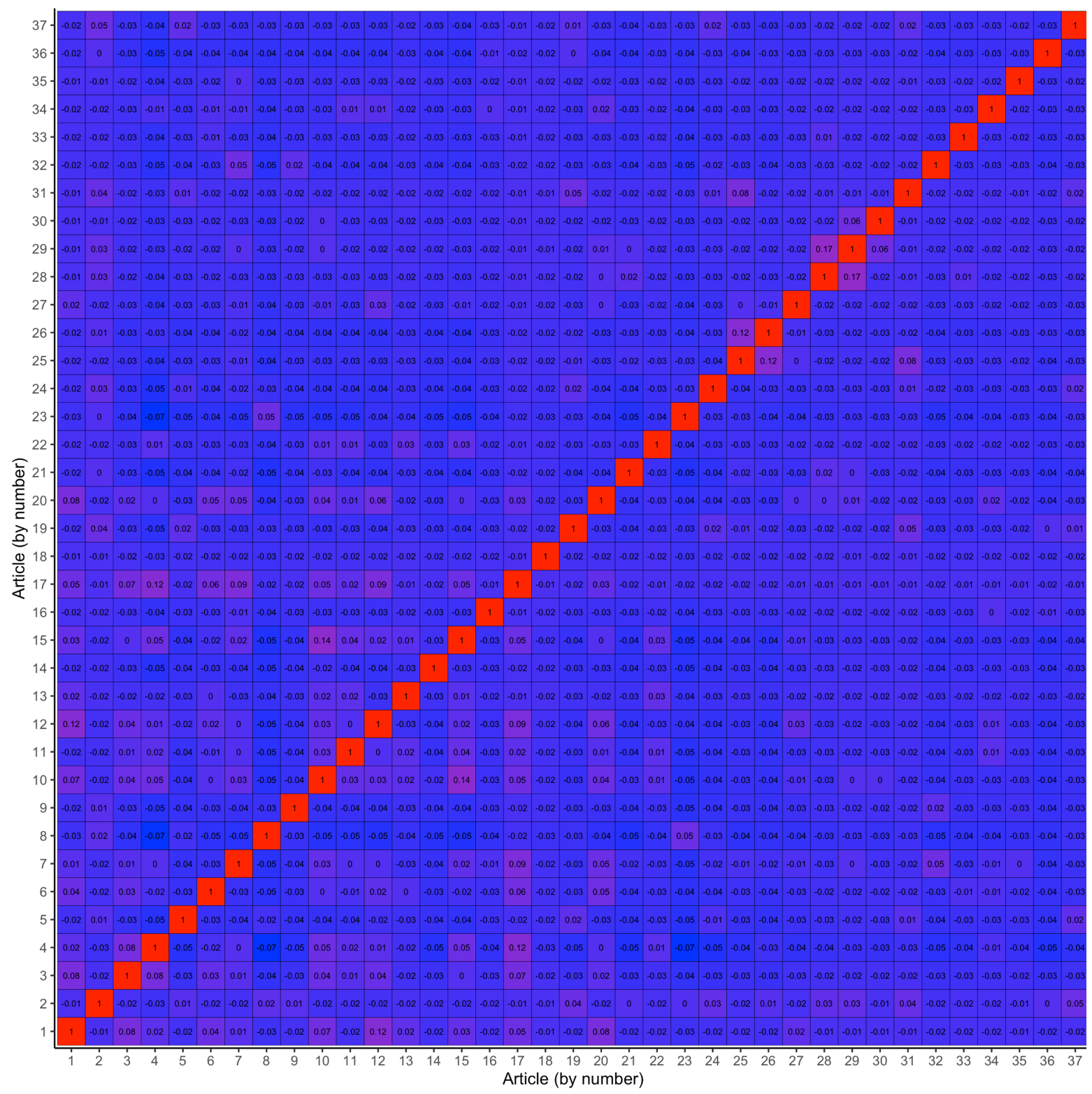

Note. The Article number refers to the bibliographic ID in the disclosure table (available online: https://doi.org/10.17605/OSF.IO/PAQ43). We were able to retrieve the full bibliographic record (including references) for 38 out of 44 articles from SCOPUS. The heatmap visualizes the overlap in referenced articles among the set of articles (Spearman rank correlation) as a rough quantitative measure of conceptual connectedness. In alignment with a qualitative coding of the investigated influence factors (see disclosure table), this quantitative analysis suggests little overlap in the conceptual structures of the included articles, undermining the breadth of the current research program on influence factors of choice consistency. 


\begin{tabular}{|c|c|c|c|}
\hline & $\begin{array}{l}\text { Binomial test } \\
\text { (Share of results } p<.025 \text { ) }\end{array}$ & $\begin{array}{l}\text { Continuous } \\
\text { (Stouffer Me }\end{array}$ & \\
\hline \multirow[t]{2}{*}{$\begin{array}{l}\text { 1) Studies contain evidential } \\
\text { value. (Right skew) }\end{array}$} & $p=.0036$ & $\begin{array}{l}\text { Full p-curve } \\
(p<.05)\end{array}$ & $\begin{array}{l}\text { Half } p \text {-curve } \\
(p<.025)\end{array}$ \\
\hline & & $\begin{array}{l}Z=-10.97 \\
p<.0001\end{array}$ & $\begin{array}{l}Z=-11.42 \\
p<.0001\end{array}$ \\
\hline $\begin{array}{l}\text { 2) Studies' evidential value, if } \\
\text { any, is inadequate. } \\
\text { (Flatter than } 33 \% \text { power) }\end{array}$ & $p=.9028$ & $\begin{array}{l}Z=7.08 \\
p>.9999\end{array}$ & $\begin{array}{l}Z=11.04 \\
p>.9999\end{array}$ \\
\hline $\begin{array}{l}\text { Statistical Power of tests } \\
\text { included in p-curve (correcting } \\
\text { for selective reporting) }\end{array}$ & \multicolumn{3}{|c|}{$\begin{array}{l}\text { Estimate: } 98 \% \\
90 \% \text { Confidence interval: }(94 \%, 99 \%)\end{array}$} \\
\hline
\end{tabular}

